\title{
See and treat strategy by LEEP conization in patients with abnormal cervical cytology
}

\author{
Fuat Demirkiran, Ilker Kahramanoglu, Hasan Turan, Nevin Yilmaz, \\ Aslihan Yurtkal, Elif Meseci, Tugan Bese, Sennur Ilvan, Macit Arvas \\ Medical Faculty, Istanbul Universit Cerrahpasa
}

\begin{abstract}
Objectives: To determine the overtreatment and re-LEEP rates of see and treat strategy (S \&T) in women who underwent S \& T by LEEP and to identify the risk factors for overtreatment and surgical margin and/or endocervical curettage positivity. Material and methods: A total of 800 patients who underwent S \& T in Istanbul University Cerrahpasa Medical Faculty between June 2010 and June 2016 were retrospectively analyzed.

Results: Overtreatment rate was found to be $46.6 \%$, decreasing with higher grade of cervical smear abnormalities. Age more than 45, low grade of cervical cytologic abnormality and absence of glandular involvement were associated with higher overtreatment rates. The more advanced the histopathology, the more increased risk of surgical margin on LEEP and ECC positivity ( $p<0.0001$, for both). Glandular involvement was associated with both surgical margin and ECC positivity.

Conclusions: S \& T can be used in patients with high grade cytologic anomaly with an acceptable overtreatment rate. In addition, bigger pieces of specimens may need to be removed during LEEP in patients who have suspicious images of higher grade of abnormalities on colposcopy to reduce surgical margin or ECC positivity. When high rate of ECC positivity in patients with HSIL cytology is considered, we suggest performing ECC to every patients with HSIL.
\end{abstract}

Key words: cervical intraepithelial neoplasia, conization, smear

Ginekologia Polska 2017; 88, 7: 349-354

\section{INTRODUCTION}

Cervical cancer remains the most common gynecological cancer around the world [1]. Due to the fact that development of cervical carcinoma occurs over a long period, which may permit an early diagnosis and treatment of precancerous cervical lesions, cytology-based screening programs have significantly reduced the incidence of the disease [2]. Women with an abnormal Pap smear result undergo colposcopic examination and colposcopic-guided biopsy, and, through this standard procedure, cervical cancer may be prevented [3]. In case of a suspicious finding for cervical intraepithelial neoplasia (CIN) 2+ on colposcopy, loop electrosurgical excision procedure (LEEP), a safe and effective treatment modality that is used in the removal of cervical dysplasia, can be offered to the patient instead of cervical biopsy for diagnosis and treatment of the disease. This option is referred to as the see and treat strategy ( $\mathrm{S} \& \mathrm{~T}$ ). There are some advantages and disadvantages of these two options for the management of women with abnormal cytology results.

S \& T has commonly been compared to the classical three step procedure involving colposcopy-guided biopsy followed by treatment of high grade lesions. The major concern of S \& T is overtreatment, which is associated with increased morbidity and cost [4]. However, management based on colposcopy-guided biopsy may be insufficient to exclude CIN 2+. Some studies have reported that, even in experienced clinics, colposcopy-guided biopsy dismisses up to $30 \%$ of high grade cervical lesion [5]. In addition, S \& T has been shown to decrease patient anxiety, number of medical visits, and travel cost [6]. It may also prevent patient dropout before treatment [7]. Some studies have reported that up to $25 \%$ of patients in the three steps strategy did not return for their next visit [8].

The last WHO guideline on screening and treatment of cervical precancerous lesions recommended the use of 
S \& T in countries where an appropriate screening strategy with cytology followed by colposcopy is present in women with ASCUS or greater results [9]. LEEP has been found to be a feasible and safe treatment method. Re-LEEP is recommended in the presence of positive surgical margin after first LEEP or positive endo-cervical curettage (ECC), as these are associated with significantly higher rates of persistence or recurrence [10].

The present study was aimed at the evaluation of S \& T for women with abnormal cytology and suspicious finding(s) on colposcopy in the diagnosis and management of cervical pathology. In this present study, we investigated the rate of overtreatment according to different abnormal cytology results. The second purpose of this study was to evaluate the factors related to positive margin and evaluate the role and importance of ECC in S \& T.

\section{MATERIAL AND METHODS}

After obtaining approval from the Ethics Committee of Istanbul University Cerrahpasa Medical Faculty, retrospective analysis of women who had undergone LEEP at the Division of Gynecologic Oncology of Cerrahpasa Medical Faculty from June 2010 to June 2016 was performed. The data were collected from the medical records. Patients' age, cervical cytology results, colposcopy findings, histopathology results of LEEP including number of specimens [one vs multiple], size of tissue, glandular involvement, and endoand ectocervical surgical margin status, histopathology results of endocervical curettage, and follow-up cytology results were consulted. Women who had undergone cervical biopsy under colposcopic examination before LEEP and those with incomplete medical records were excluded.

Women with cytological abnormalities or post-coital bleeding and women with suspicious macroscopic findings on vaginal examination underwent colposcopy. An experienced gynecologic oncologist or a trained fellow of gynecologic oncology performed all examinations. A Carl Zeiss colposcope (Carl Zeiss. Oberkochen, Germany) with a magnification of between $4 \times$ and $20 \times$ was used for colposcopy. Subsequently, patients were offered the option of LEEP when CIN 2+ was suspected or colposcopic examination was inadequate. A 15 to $25 \mathrm{~mm}$ round loop electrode was used at $60 \mathrm{~W}$ aimed at excision of the transformation zone or the lesion. ECC was performed with a size 0 uterine curette in all patients following LEEP. Hemostasis was obtained by cauterization of the surgical site using a ball electrode at a setting of $50 \mathrm{~W}$. The pieces were fixed in formalin and sent for histopathologic evaluation. All of the LEEP and ECC procedures were performed by gynecologic oncology fellows or by trained physicians, supervised by gynecologic oncology fellows. All of the specimens were evaluated by the same pathologist (S.I.).
The cervical cytology results were classified in accordance with the Bethesda System of 2001 [11]. The histological findings were described as negative, cervical intraepithelial lesion 1, CIN 2, CIN 3, adenocarcinoma in situ (AIS), or cancer. CIN 2+ was defined as CIN 2-3, AIS, and cancer. Overtreatment was defined as having CIN 1 or a negative result on final histopathology.

Re-LEEP was offered to patients if positive surgical margin after LEEP or pre-invasive pathology at ECC was present. After obtaining negative surgical margins, post-LEEP follow-up was every six months for one year by cervical cytology, and then annually if two subsequent normal cytological results were obtained. Recurrence was defined as having ASCUS, LSIL, ASC-H, HSIL, or atypical glandular cells on cervical cytology during follow-up.

Statistical analyses were performed using Statistical Package for the Social Sciences (SPSS) version 16.0 (SPSS Inc., Chicago, IL, USA), while clinicopathological variables, including the categorical data, were analyzed using the chi-square or Fisher's exact test. All reported confidence interval (Cl) values were calculated at the $95 \%$ level. A probability value of less than 0.05 was defined as statistically significant.

\section{RESULTS}

Clinicopathologic characteristics of the patients are presented in Table 1. The women had a mean age of 34.1 years, with a range of 19-68. LSIL and HSIL were the most common cytological anomalies managed with S \& T. Additionally, 17 women had a normal Pap smear result, 14 had post-coital

Table 1. Clinicopathologic characteristics of study group

\begin{tabular}{|c|c|c|}
\hline & Mean (year) & Range (year) \\
\hline Age & 34.1 & $19-68$ \\
\hline Pap smear & $\mathbf{n}$ & $\%$ \\
\hline Negative & & \\
\hline ASCUS & 17 & 2.1 \\
\hline LSIL & 130 & 16.2 \\
\hline ASC-H & 280 & 35.0 \\
\hline HSIL & 109 & 13.6 \\
\hline AGC & 244 & 30.5 \\
\hline Final histology & 20 & 2.5 \\
\hline Negative & 171 & \\
\hline CIN I & 202 & 21.3 \\
\hline CIN II & 105 & 25.2 \\
\hline CIN III & 302 & 13.1 \\
\hline AIS & 2 & 37.7 \\
\hline Cancer & 18 & 0.2 \\
\hline Total & 800 & 2.2 \\
\hline
\end{tabular}


bleeding, and 3 had macroscopic cervical lesions. Thus, they underwent colposcopic examination and subsequent management with $\mathrm{S} \& \mathrm{~T}$. While pathological results of LEEP specimens were CIN 2+ in 427 out of 800 patients (53.3\%), 373 patients had CIN I or negative pathology, reflecting an overtreatment rate of $46.6 \%$.

When histopathological results after LEEP were evaluated per cytology results, 32.3\%, 37.8\%, 65.1\%, $82.3 \%$, and $35 \%$ of women with ASCUS, LSIL, ASC-H, HSIL, and AGC cytology results, respectively, had CIN 2+ on final pathology (Table 2). The rate of CIN2+ lesion was $5.8 \%$ in women with normal cervical cytology. Cervical cancer was detected in four of 130 (3\%) patients with ASCUS cytology. Furthermore, $2.8 \%$ and $4.5 \%$ of patients with ASCH and HSIL cytology had cervical cancer after LEEP, respectively.

Overtreatment rates according to cytology results are presented in Table 3. No statistically significant relation was identified between unsatisfactory colposcopic examination

Table 2. Histological results of LEEP specimens, according to cytology results

\begin{tabular}{|l|c|c|c|c|c|c|}
\hline \multirow{2}{*}{ Histology } & \multicolumn{7}{|c|}{ Pap smear } \\
\cline { 2 - 8 } & $\begin{array}{c}\text { Negative } \\
\mathbf{n}(\%)\end{array}$ & $\begin{array}{c}\text { ASCUS } \\
\mathbf{n}(\%)\end{array}$ & $\begin{array}{c}\text { LSIL } \\
\mathbf{n}(\%)\end{array}$ & $\begin{array}{c}\text { ASC-H } \\
\mathbf{n}(\%)\end{array}$ & $\begin{array}{c}\text { HSIL } \\
\mathbf{n}(\%)\end{array}$ & $\begin{array}{c}\text { AGC } \\
\mathbf{n}(\%)\end{array}$ \\
\hline Negative & $10(58.8)$ & $54(41.5)$ & $65(23.2)$ & $22(20.1)$ & $9(3.6)$ & $11(55)$ \\
\hline CIN I & $6(35.2)$ & $34(26.1)$ & $110(39.2)$ & $16(14.6)$ & $34(13.9)$ & $2(10)$ \\
\hline CIN II+ & $1(5.8)$ & $42(32.3)$ & $105(37.5)$ & $71(65.1)$ & $201(82.4)$ & $7(35)$ \\
\hline CIN II & - & $13(10)$ & $44(15.7)$ & $12(11)$ & $36(14.7)$ & - \\
\hline CIN III & $1(5.8)$ & $25(19.2)$ & $61(21.7)$ & $55(50.4)$ & $153(62.7)$ & $7(35)$ \\
\hline AIS & - & - & - & $1(0.9)$ & $1(0.4)$ & - \\
\hline Cancer & - & $4(3)$ & - & $3(2.8)$ & $11(4.5)$ & - \\
\hline Total & $\mathbf{1 7}(\mathbf{1 0 0}$ & $\mathbf{1 3 0 ( 1 0 0 )}$ & $\mathbf{2 8 0 ( 1 0 0 )}$ & $\mathbf{1 0 9 ( 1 0 0 )}$ & $\mathbf{2 4 4}(\mathbf{1 0 0})$ & $\mathbf{2 0 ( 1 0 0 )}$
\end{tabular}

Table 3. Possible risk factors for overtreatment in S \& T

\begin{tabular}{|c|c|c|c|c|}
\hline & $\mathbf{n}$ & $\begin{array}{l}\text { Overtreatment }(n=373) \\
n(\%)\end{array}$ & $\begin{array}{l}\text { Proper treatment }(n=427) \\
\qquad n(\%)\end{array}$ & p value \\
\hline \multicolumn{4}{|l|}{ Colposcopy } & \multirow{3}{*}{ NS } \\
\hline Satisfactory & 532 & $238(44.7)$ & $294(55.2)$ & \\
\hline Unsatisfactory & 268 & $135(50.3)$ & $133(49.6)$ & \\
\hline \multicolumn{4}{|l|}{ Age (years) } & \multirow{3}{*}{$<0.0001$} \\
\hline$\leq 45$ & 618 & $263(42.5)$ & $355(57.4)$ & \\
\hline$>45$ & 182 & $110(60.4)$ & $72(39.5)$ & \\
\hline \multicolumn{4}{|l|}{ Initial cytology } & \multirow{7}{*}{$<0.0001$} \\
\hline Negative & 17 & $16(94.1)$ & $1(5.8)$ & \\
\hline ASCUS & 130 & $88(67.6)$ & $42(32.3)$ & \\
\hline LSIL & 280 & $175(62.5)$ & $105(37.5)$ & \\
\hline ASC-H & 109 & $38(34.8)$ & $71(65.1)$ & \\
\hline HSIL & 244 & $43(17.6)$ & $201(82.4)$ & \\
\hline AGC & 20 & $13(65)$ & $7(35)$ & \\
\hline \multicolumn{4}{|c|}{ Number of fragments of LEEP } & \multirow{3}{*}{ NS } \\
\hline Single & 680 & $323(47.5)$ & $357(52.5)$ & \\
\hline Multiple & 120 & $50(41.6)$ & $70(58.3)$ & \\
\hline \multicolumn{4}{|c|}{ Glandular involvement } & \multirow{3}{*}{$<0.0001$} \\
\hline Negative & 660 & $365(55.3)$ & $295(44.7)$ & \\
\hline Positive & 140 & $8(5.7)$ & $132(94.3)$ & \\
\hline
\end{tabular}

NS - not significant 
Table 4. Relationship between clinicopathologic factors and surgical margin ${ }^{2}$

\begin{tabular}{|c|c|c|c|c|c|c|}
\hline & \multicolumn{2}{|c|}{ Surgical margin } & \multirow[b]{2}{*}{ p value } & \multicolumn{2}{|c|}{ ECC } & \multirow[b]{2}{*}{$p$ value } \\
\hline & $\begin{array}{c}(+) \\
n(\%)\end{array}$ & $\begin{array}{c}(-) \\
n(\%)\end{array}$ & & $\begin{array}{c}(+) \\
\text { n(\%) }\end{array}$ & $\begin{array}{c}(-) \\
n(\%)\end{array}$ & \\
\hline \multicolumn{6}{|l|}{ Initial cytology } & \multirow{6}{*}{$<0.0001$} \\
\hline ASCUS & $16(12.3)$ & $114(87.6)$ & \multirow{5}{*}{$<0.0001$} & $5(3.8)$ & $125(96.1)$ & \\
\hline LSIL & $35(12.5)$ & $245(87.5)$ & & $5(1.7)$ & 275 (98.2) & \\
\hline ASC-H & $28(25.6)$ & $81(74.3)$ & & $7(6.4)$ & $102(93.5)$ & \\
\hline HSIL & $98(40.1)$ & $146(59.8)$ & & 25 (20.4) & 219 (79.5) & \\
\hline AGC & $2(10)$ & $18(90)$ & & $1(5)$ & $19(95)$ & \\
\hline \multicolumn{3}{|l|}{ Colposcopy } & \multirow{3}{*}{ NS } & & & \multirow{3}{*}{ NS } \\
\hline Satisfactory & $128(29.6)$ & $304(70.3)$ & & $31(7.1)$ & $403(92.8)$ & \\
\hline Unsatisfactory & $51(28.4)$ & $146(32.4)$ & & $12(6)$ & $185(93.9)$ & \\
\hline \multicolumn{3}{|l|}{ Age (years) } & \multirow{3}{*}{ NS } & & & \multirow{3}{*}{$<0.0001$} \\
\hline$\leq 45$ & $141(27.2)$ & $375(72.7)$ & & $21(4)$ & $495(95.9)$ & \\
\hline$>45$ & $38(33.6)$ & $75(66.3)$ & & $22(19.1)$ & $93(80.9)$ & \\
\hline \multicolumn{3}{|c|}{ Number of fragments of LEEP } & \multirow{3}{*}{ NS } & & & \multirow{3}{*}{ NS } \\
\hline Single & $145(27)$ & $391(73)$ & & $33(6.1)$ & $505(93.8)$ & \\
\hline Multiple & $34(36.5)$ & $59(63.5)$ & & $10(10.7)$ & $83(89.3)$ & \\
\hline \multicolumn{3}{|l|}{ Histopathology } & \multirow{3}{*}{$<0.0001$} & & & \multirow{3}{*}{$<0.0001$} \\
\hline CIN I & $13(6.4)$ & $189(93.5)$ & & $1(0.4)$ & $201(99.5)$ & \\
\hline CIN II+ & $166(38.8)$ & $261(61.1)$ & & $42(9.7)$ & $387(90.2)$ & \\
\hline \multicolumn{3}{|c|}{ Glandular involvement } & \multirow{3}{*}{$<0.0001$} & & & \multirow{3}{*}{$<0.0001$} \\
\hline Negative & $92(18.6)$ & $399(81.3)$ & & $14(2.8)$ & $478(97.1)$ & \\
\hline Positive & $87(63)$ & $51(36.9)$ & & 29 (20.8) & 110 (79.1) & \\
\hline Total & 179 & 450 & & 43 & 588 & \\
\hline
\end{tabular}

ane hundred and seventy one women with negative histology were excluded from analysis. Two women had negative LEEP but positive ECC result. Finally, a total of 629 and 631 women were analysed for surgical margin and ECC status, respectively; NS — not significant

and overtreatment rate. However, age $>45$, initial cytology, and glandular involvement were found to be significantly associated with overtreatment rate.

Table 4 shows the factors related to positive surgical margin and ECC. Initial cytology result, histolopathology, and glandular involvement were associated with both surgical margin and ECC positivity. Age more than 45 years was associated with ECC positivity, but not with surgical margin positivity. The more advanced the histopathology, the more increased risk of surgical margin on LEEP and ECC positivity ( $p<0.0001$, for both). The rates of positive margin were $6.4 \%$ and $43.3 \%$ in patients having CIN I and CIN III diseases, respectively. Only $0.4 \%$ of patients with CIN I had pre-invasive disease in the endocervix; however, the ratio was $9.5 \%$ for patients with CIN III. In the whole group, positive margin rate and ECC positivity were $22.3 \%$ and $5.3 \%$, respectively. Out of 179 patients who had positive surgical margin, 90 had endocervical margin positivity, 45 had ectocervical margin positivity, and 44 had both endo- and ectocervical margin positivity.
Most of the patients were regularly followed-up (697 of 800 patients; $87.1 \%$ ). When 122 patients who had undergone re-LEEP because of surgical margin or ECC positivity were excluded, recurrence was observed in 24 of 575 patients (4.1\%) within 31 months mean follow-up time.

\section{DISCUSSION}

In the present study, the rates of CIN II+ lesions were 32\%, $37 \%, 65 \%$, and $82 \%$ in patients with ASCUS, LSIL, ASC-H, and HSIL cytology results, respectively. These high CIN II + lesion rates are not compatible with many studies. In their study, Cho et al. [12] found that 26 of 145 (18\%) patients with low grade cervical smear had undergone $S \& T$ due to high grade colposcopic impression had CIN II+ in their final pathology. The only study including 58 ASC-H patients who had undergone $S \& T$ found a $72 \%$ rate of CIN II+ in their LEEP results [13]. CIN II+ rate was found to be between $65 \%$ and $90 \%$ in HSIL cytologies when managed according to $S \& T$ [14].

Increased high-grade lesion rates even in minimal abnormal cytology such as ASCUC and LSIL may be based on 
the management of these patients in a single gynecologic oncology clinic and the fact that many of the patients are referred from other clinics. With these findings, we can say that in contrast to the new recommendations of some societies, all women with ASCUS should be evaluated by colposcopic examination for the diagnosis of CIN II + lesion including cervical cancer [15].

The most problematic issue related to $S \& T$ is the high overtreatment rate of this approach for the management of the women who present with minimal abnormal cytology (ASCUS or LSIL) results. In this group of patients, the overtreatment rate was found to be $65 \%$. When S \& T has been applied to patients with low grade cytology, the overtreatment rate may rise up to $60 \%$ [16]. In a recent meta-analysis, the overtreatment rate was found to be $46 \%$ in patients with ASCUS or LSIL [14]. Overtreatment rates of S \& T and three-step procedure in high grade abnormal cytologies are comparable considering the $11-35 \%$ rates of overtreatment of cervical biopsy under colposcopy followed by LEEP if the biopsy shows CIN 2+ [16, 17]. In addition, our findings are compatible with the recommendation of American Society for Colposcopy and Cervical Pathology, which states that it is acceptable to treat women with HGSIL cytology according to S\&T, except those who are pregnant or aged 21-24 years [18]. In the meantime, considering the recommendation of the British Society for Colposcopy and Cervical Pathology, which states that overtreatment rate should be under 10\%, S \& T may be abandoned for low grade cytology [19]. According to the results of this present study, S \& T does not make sense for patients with minimal abnormal cytology because of high overtreatment rate, but it is logical in the case of HSIL cytology.

There are some factors that affect the overtreatment rate of S \& T protocol. For example, Bosgraaf et al. [20] demonstrated that women aged 40 years or more are more likely to be over-treated. Additionally, Kim YJ et al. [21] found that ASCUS and LSIL cytology are associated with higher rates of overtreatment in patients who undergo $S \& T$. In our study, overtreatment was seen in only $6 \%$ of patients with glandular involvement. Also, in the advanced age group, overtreatment was much higher compared to the other group ( $<45$ years of age) in the study. The main reason for this is physicians prefer $S \& T$ rather than the three-step treatment for advanced age patients due to insufficient colposcopy results. Thus, postmenopausal patients should be selected more carefully for $\mathrm{S} \& \mathrm{~T}$. As glandular involvement can be determined at final pathology, it cannot be used for selection of patients for $\mathrm{S} \& \mathrm{~T}$.

Positive surgical margin following LEEP has been shown to be associated with relapse or progression of the disease [10]. A meta-analysis of 35.100 patients with any grade of CIN or cervical cancer demonstrated a $23 \%$ rate of positive surgical margin following conization [22]. Age more than 50 years, ECC positivity, gravidity $>2$, high risk HPV load, and parity $>4$ were demonstrated as significant factors for incomplete excision [23]. In our series, $22.3 \%$ of patients who had undergone LEEP for $\mathrm{S} \& \mathrm{~T}$ had positive surgical margin. Our study demonstrated that the rate of surgical margin positivity gradually increases with the degree of abnormality in the cervical smear. While almost $12 \%$ of patients with minimal cytological abnormality had positive surgical margin after LEEP, the ratio was $40 \%$ for patients with HSIL. Similarly, ECC positivity increased with the severity of abnormal cytology. Twenty percent of patients with HSIL cytology had ECC positivity. In addition, we showed that glandular involvement increases the risk of surgical margin positivity as well as higher grade of abnormality. Consistent with our results, Ayhan et al. found that CIN 2+ on final pathology is associated with surgical margin positivity [24]. They also showed that the need for multiple sweeps during LEEP was the most important predictor of disease persistence [24]. We know that LEEP is associated with higher rates of positive surgical margin in lesions involving more than two-third of the cervix compared to cold-knife conization [25]. As an indicator of number of sweeps, the number of fragments of LEEP was not associated with surgical margin positivity in our study. One possible explanation for different results among both studies may be that a 15-20 mm round loop electrode was used in their study, while we used a 15 to $25 \mathrm{~mm}$ round loop according to cervical dimension. From our point of view, use of larger loops or cold-knife excision may be helpful to provide clear surgical margins.

In the present study, 179 of 629 patients with CIN I or higher in final pathology had surgical margin positivity (28.4\%). In our clinic, re-LEEP is offered to patients with positive surgical margin or ECC. Regular follow-up for each six month period in the first year and then annually are performed for patients who have undergone LEEP. Even though $17 \%$ rate of recurrence was reported in patients with negative surgical margin after LEEP was performed, only $4 \%$ of our patients with no surgical margin involvement recurred within 31 months' follow-up [3]. This low recurrence rate may be related to the short follow-up period.

The major strengths of our study are the large number of patients and the fact that the same gynecopathologists evaluated the LEEP specimens. The main limitations are its retrospective design and that Pap smears were evaluated by different pathologists within different pathology laboratories. These limitations may explain the high rate of cancer in ASCUS cytology.

In conclusion, our findings suggest that the see and treat strategy can be used in patients with high grade cytologic anomaly with an acceptable overtreatment rate, regardless of age. Patients with low grade cytologic abnormalities 
should be counselled about high rates of overtreatment. $S \& T$ may be beneficial in those who are prone to dropout from treatment, especially in patients less than 45 years of age. In addition, bigger pieces of specimens may need to be removed during LEEP in patients who have suspicious images of higher grade abnormalities on colposcopy to reduce surgical margin or ECC positivity. Given the high rate of ECC positivity in patients with HSIL cytology, we suggest performing ECC on every patient with HSIL during LEEP.

\section{Funding}

None.

\section{Conflict of interest}

Authors declare no conflict of interest.

\section{Ethical approval}

This article does not contain any studies with human participants or animals performed by any of the authors. Ethics Committee of Istanbul University Cerrahpasa Medical Faculty approved the study protocol.

\section{REFERENCES}

1. Ferlay J, Soerjomataram I, Ervik M, et al. GLOBOCAN 2012 v1. 0, Cancer Incidence and Mortality Worldwide: IARC CancerBase No. 11.

2. Smith HJ, Leath CA, Huh WK, et al. See-and-treat for high-grade cytology: do young women have different rates of high-grade histology? J Low Genit Tract Dis. 2016; 20(3): 243-246, doi: 10.1097/LGT.0000000000000229, indexed in Pubmed: 27243140.

3. Nogara PRB, Manfroni LAR, da Silva MC, et al. The "see and treat" strategy for identifying cytologic high-grade precancerous cervical lesions among low-income Brazilian women. Int J Gynaecol Obstet. 2012; 118(2): 103-106, doi: 10.1016/j.ijgo.2012.02.017, indexed in Pubmed: 22608027.

4. Massad LS. New guidelines on cervical cancer screening: more than just the end of annual Pap testing. J Low Genit Tract Dis. 2012; 16(3): 172-174, doi: 10.1097/LGT.0b013e31824bc178, indexed in Pubmed: 22820979.

5. Moss EL, Hadden P, Douce G, et al. Is the colposcopically directed punch biopsy a reliable diagnostic test in women with minor cytological lesions? J Low Genit Tract Dis. 2012; 16(4): 421-426, doi: 10.1097/LGT.0b013e318250acf3, indexed in Pubmed: 22669079.

6. Chigbu CO, Onyebuchi AK. See-and-treat management of high-grade squamous intraepithelial lesions in a resource-constrained African setting. Int J Gynaecol Obstet. 2014; 124(3): 204-206, doi: 10.1016/j. ijgo.2013.07.040, indexed in Pubmed: 24377647.

7. Monteiro AC, Russomano F, Reis A, et al. Effectiveness of see-and-treat for approaching pre-invasive lesions of uterine cervix. Rev Saude Publica. 2009; 43(5): 846-850, indexed in Pubmed: 19851632.

8. Lindeque BG. Management of cervical premalignant lesions. Best Pract Res Clin Obstet Gynaecol. 2005; 19(4): 545-561, doi: 10.1016/j. bpobgyn.2005.02.008, indexed in Pubmed: 16150393.

9. World Health Organization. WHO guidelines for screening and treatment of precancerous lesions for cervical cancer prevention. http://www.who. int/reproductivehealth/publications/cancers/screening and treatment_of_precancerous_lesions/en/index.html.Updated 2013. Accessed 01.12.2016.

10. Wong ASM, Li WH, Cheung TH. Predictive factors for residual disease in hysterectomy specimens after conization in early-stage cervical cancer.
Eur J Obstet Gynecol Reprod Biol. 2016; 199: 21-26, doi: 10.1016/j. ejogrb.2016.01.020, indexed in Pubmed: 26894378.

11. Solomon D, Davey D, Kurman R, et al. Forum Group Members, Bethesda 2001 Workshop. The 2001 Bethesda System: terminology for reporting results of cervical cytology. JAMA. 2002; 287(16): 2114-2119, indexed in Pubmed: 11966386.

12. Cho H, Kim JH. Treatment of the patients with abnormal cervical cytology: a "see-and-treat" versus three-step strategy. J Gynecol Oncol. 2009; 20(3): 164-168, doi: 10.3802/jgo.2009.20.3.164, indexed in Pubmed: 19809550.

13. Kietpeerakool C, Cheewakriangkrai C, Suprasert P, et al. Feasibility of the 'see and treat' approach in management of women with 'atypical squamous cell, cannot exclude high-grade squamous intraepithelial lesion' smears. J Obstet Gynaecol Res. 2009; 35(3): 507-513, doi: 10.1111/j.1447-0756.2008.00992.x, indexed in Pubmed: 19527391.

14. Ebisch RMF, Rovers MM, Bosgraaf RP, et al. Evidence supporting see-and-treat management of cervical intraepithelial neoplasia: a systematic review and meta-analysis. BJOG. 2016; 123(1):59-66, doi: 10.1111/14710528.13530, indexed in Pubmed: 26177672.

15. Massad LS, Einstein MH, Huh WK, et al. 2012 ASCCP Consensus Guidelines Conference, 2012 ASCCP Consensus Guidelines Conference. 2012 updated consensus guidelines for the management of abnormal cervical cancer screening tests and cancer precursors. J Low Genit Tract Dis. 2013; 17(5 Suppl 1): S1-S27, doi: 10.1097/LGT.0b013e318287d329, indexed in Pubmed: 23519301.

16. Guldeniz AD, Turkan $G$, Murat $B C$. Is the loop electrosurgical excision procedure necessary for minor cervical cytological abnormalities? Asian Pac J Cancer Prev. 2014; 15(1): 305-308, indexed in Pubmed: 24528046.

17. Li ZG, Qian DeY, Cen JM, et al. Three-step versus "see-and-treat" approach in women with high-grade squamous intraepithelial lesions in a low-resource country. Int J Gynaecol Obstet. 2009; 106(3): 202-205, doi: 10.1016/j.ijgo.2009.04.011, indexed in Pubmed: 19477446.

18. Massad LS, Einstein MH, Huh WK, et al. 2012 ASCCP Consensus Guidelines Conference, 2012 ASCCP Consensus Guidelines Conference. 2012 updated consensus guidelines for the management of abnormal cervical cancer screening tests and cancer precursors. J Low Genit Tract Dis. 2013; 17(5 Suppl 1): S1-S27, doi: 10.1097/LGT.0b013e318287d329, indexed in Pubmed: 23519301.

19. Luesley D, Leeson S. Colposcopy and Programme Management. Guidelines for the NHS Cervical Screening Programme. NHSCSP Publication, no. 20. Sheffield, UK: NHSCSP; 2010.

20. Bosgraaf RP, Mast PP, Struik-van der Zanden PH, et al. Overtreatment in a see-and-treat approach to cervical intraepithelial lesions. Obstet Gynecol. 2013; 121(6): 1209-1216, doi: 10.1097/AOG.0b013e318293ab22, indexed in Pubmed: 23812454.

21. Kim JiYe, Lee $\mathrm{DH}$, Kang JH, et al. The overtreatment risk of see-and-treat strategy in management of abnormal cervical cytology. Gynecol Obstet Invest. 2014; 78(4): 239-243, doi: 10.1159/000365476, indexed in Pubmed: 25341379.

22. Ghaem-Maghami S, Sagi S, Majeed G, et al. Incomplete excision of cervical intraepithelial neoplasia and risk of treatment failure: a meta-analysis. Lancet Oncol. 2007; 8(11): 985-993, doi: 10.1016/S14702045(07)70283-8, indexed in Pubmed: 17928267.

23. Park JY, Lee KH, Dong SM, et al. The association of pre-conization high-risk HPV load and the persistence of HPV infection and persistence/recurrence of cervical intraepithelial neoplasia after conization. Gynecol Oncol. 2008; 108(3):549-554, doi: 10.1016/j.ygyno.2007.11.009, indexed in Pubmed: 18177929.

24. Ayhan A, Tuncer HA, Reyhan NH, et al. Risk factors for residual disease after cervical conization in patients with cervical intraepithelial neoplasia grades 2 and 3 and positive surgical margins. Eur J Obstet Gynecol Reprod Biol. 2016; 201: 1-6, doi: 10.1016/j.ejogrb.2016.03.021, indexed in Pubmed: 27038228

25. Chen $Y, L u ~ H$, Wan X, et al. Factors associated with positive margins in patients with cervical intraepithelial neoplasia grade 3 and postconization management. Int J Gynaecol Obstet. 2009; 107(2): 107-110, doi: 10.1016/j.ijgo.2009.05.027, indexed in Pubmed: 19628208. 